\title{
Phase Shift Migration for Imaging Layered Objects and Objects Immersed in Water
}

\author{
Tomas Olofsson
}

\begin{abstract}
This paper proposes the use of phase shift migration for ultrasonic imaging of layered objects and objects immersed in water. The method, which was developed in reflection seismology, is a frequency domain technique that in a computationally efficient way restores images of objects that are isotropic and homogeneous in the lateral direction but inhomogeneous in depth. The performance of the proposed method was evaluated using immersion test data from a block with side-drilled holes with an additional scatterer residing in water. In this way, the method's capability of simultaneously imaging scatterers in different media and at different depths was investigated. The method was also applied to a copper block with flat bottom holes. The results verify that the proposed method is capable of producing high-resolution and lownoise images for layered or immersed objects.
\end{abstract}

\section{INTRODUCTION}

$\mathrm{O}$ NE important imaging method in ultrasonic testing is the synthetic aperture focusing technique (SAFT), which was introduced in ultrasonic nondestructive testing (NDT) in the early 1970s [1] and has been in wide use since the late 1980s. In basic SAFT, a synthetic array is emulated in post-processing using pulse-echo data acquired with a physically scanned single-element transducer. The post-processing consists of delay-and-sum beamforming and the resulting images generally have far better lateral resolution than the B-scans from which they are constructed. Moreover, the lateral resolution is independent of depth. The coherent summation also yields an increase in signal-to-noise ratio resulting in an improved depth-of-field.

The early versions of SAFT were time domain implementations, followed later by implementations in the frequency domain [2]-[5] evolving from acoustical holography $[6]$ and diffraction tomography. The frequency domain implementations are particularly attractive if the sound speed is constant throughout the entire propagation medium, something which holds when a homogeneous and isotropic object is tested in contact. Then SAFT can be implemented in a computationally very efficient form using a method that was first proposed in geophysics [7], called Stolt migration or the frequency wavenumber method [3], [4], [8].

Practical drawbacks with contact testing are transducer wear and potentially poor or nonuniform coupling between transducer and test object caused by rough sur-

Manuscript received February 2, 2010; accepted May 24, 2010.

The author is with the Department of Engineering Sciences, Uppsala University, Uppsala, Sweden (e-mail: tomas.olafsson@signal.uu.se).

Digital Object Identifier 10.1109/TUFFC.2010.1718 faces and, therefore, immersion testing is often preferred in NDT. Note that the assumption of constant sound speed in the propagating medium no longer holds for immersion testing because the propagation involves at least two sound speeds, one in water and one in the solid. Thus, unfortunately, the conditions required by the frequency wavenumber method do not hold. In time domain SAFT, the problem manifests itself in that the refraction at the liquid-solid interface complicates the computation of the travel times required in the algorithm. Such complications are, of course, present in any situation having test objects consisting of layers with different sound speeds.

Despite these difficulties, which to some extent have prevented the use of SAFT for immersion test data, various methods have been proposed to treat the immersion case. One such method, which is restricted to one refracting layer only, is to use transducers that are focused on the object's surface, see e.g., [9], in this way emulating a scenario similar to contact testing with a transducer having a diameter of the size of the focal diameter. Note, however, that diffraction causes the acoustical field emitted from a focused transducer to take a form that may deviate from the spherical wave anticipated from a small transducer [10], thus to some extent violating the assumptions on which SAFT are based and thereby limiting the usefulness of the approach.

More generally, in inhomogeneous materials, the travel times required in time domain SAFT can be calculated using ray-tracing techniques [11]-[13] that are typically time consuming, thus limiting their practical usefulness. For the special case of horizontally layered materials, travel time approximations can be obtained more efficiently by means of so-called root-mean-squared (RMS) velocities [14], provided that the sound speed differences between layers are relatively small. This holds in many cases in reflection seismology, for which it is often used, and in medical ultrasound where it has recently been used in the context of aberration correction [15]. When testing metals in immersion, the sound speed contrast is typically high and the RMS-velocity approach has thus far not been used in the NDT community.

In reflection seismology, the case of objects consisting of horizontal homogenous layers has been treated extensively. There, a method called phase shift migration [16] has been developed for treating this scenario. Together with Stolt migration, it has become an important tool for analyzing the Earth's interior structure [17] and it has been generalized in different ways, for instance, to admit also some lateral variations in sound speed [18]. Phase shift migration is a frequency domain approach that makes 
explicit use of the wave equation in the processing. Viewing the measured signals at the sensors as a boundary condition for the wave equation, the field is extrapolated using a phase-shift operator in the frequency domain to hypothetical measurements of the field at a set of different depths. From these, the image can be obtained in a fairly straightforward manner, see Section II.

Because of its iterative nature, the method is computationally less efficient than Stolt migration but, on the other hand, phase shift migration can treat the more complicated scenario of having a sound speed that is constant in the lateral direction but varying in depth.

In this work, we investigate phase shift migration for imaging data acquired using immersion testing, which is an application that is particularly well-suited for phase shift migration because the sound speeds necessary in the processing typically are known to a high accuracy. The application used for illustration is immersion testing of copper blocks using a single scanned transducer and the experiments aim at demonstrating the potential of phase shift migration for this application. Note, however, that there are other potential important applications of the method. One such is inspection, in contact or in immersion, of metal objects with a protective stainless steel overlay having a different sound speed than the backing material.

Phase shift migration has been previously used for ultrasonic imaging in [19]. Furthermore, the image-formation equations in [5] are essentially identical to those in phase shift migration. However, the potential of allowing vertical sound speed variations was not exploited in either of these two references. This was done in [6], but the imaging was performed using monochromatic signals.

In this paper, we limit the work to concern only 2-D migration but we note that full 3-D migration is a straightforward extension. The theory and the algorithm are given in Section II. Experiments illustrating the approach are presented in Section III and, finally, conclusions and a discussion are given in Section IV.

\section{THEORY}

In this section, a derivation of the reconstruction algorithm used in the work is given. The derivation presented in Section II-A is based on what, in geophysics, is traditionally called the exploding reflector model [17], [20]. The reader should note that this model does not describe the single-transducer scanned pulse-echo measurements scenario, hereafter called the monostatic ${ }^{1}$ case; rather, it is an analogous physical scenario for which a simple imaging algorithm can be developed. The idea is thus to solve the imaging problem for this simple case and then show how the monostatic case can be transformed to it by simple means.

\footnotetext{
${ }^{1}$ The term monostatic is borrowed from the radar community.
}

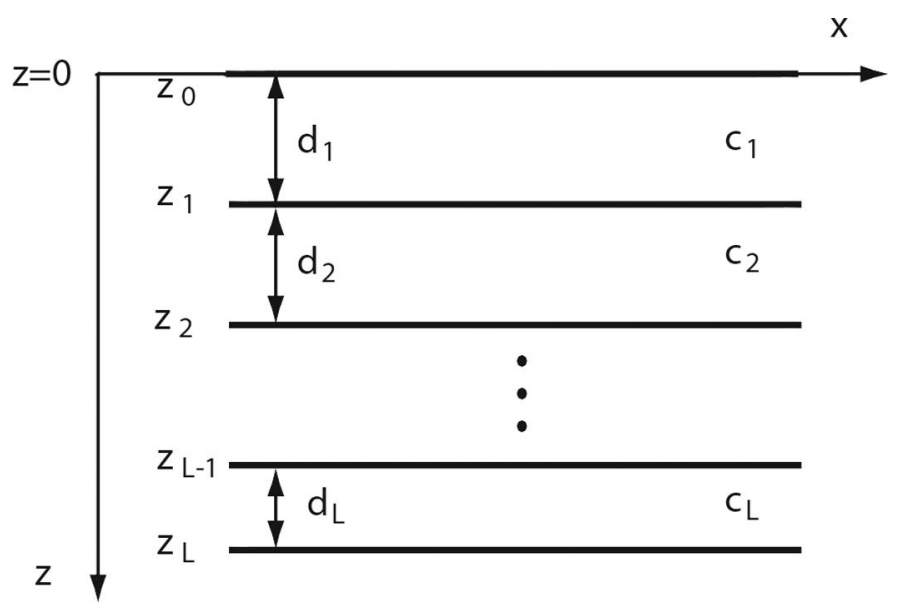

Fig. 1. The layered media considered in the derivation.

In the exploding reflector model, the reflectors in the medium are assumed to "explode" simultaneously at time $t=0$, each with a strength that is proportional to its reflectivity, creating a field that is simultaneously measured using an array of sensors. The model neglects multiple reflections between layers and only upward-traveling waves are considered. Furthermore, transmission losses at layer interfaces are neglected; see Appendix A for comments on this.

The derivation deviates from those found in the classical references, such as [16], in the respect that the vertical sound speed variations are introduced early in the model and not appearing as a last modification of a solution developed for a medium with constant sound speed; see [21] for comments on such inconsistencies.

Section II-B describes the steps necessary for adapting the monostatic case to an analogous exploding reflector scenario. A summary of the algorithm is given in Section II-C.

\section{A. Phase Shift Migration for the Exploding Reflector Scenario}

In the following, we consider propagation of longitudinal waves in a medium illustrated in Fig. 1. It consists of $L$ horizontal isotropic layers with thicknesses $d_{1}, \ldots, d_{L}$ and sound speeds, $c_{1}, \ldots, c_{L}$, respectively. The coordinate axes $x$ and $z$ point in the horizontal and vertical directions, respectively, with $z$ pointing downwards. We let $z_{l}$ denote the $z$-coordinate of the lower side of layer $l$, i.e., $z_{l}=$ $\sum_{q=1}^{l} d_{q}$.

Let $p(x, z, t)$ denote the field resulting from the reflectors in the media exploding simultaneously at $t=0$. This field is observed along the horizontal line $z=0$ and our aim is to extrapolate this observed field to lines at other depths within the media. As will be described later, these can then be used straightforwardly to create an image of the media.

The field extrapolation can be done by decomposing the field into a set of upward-traveling harmonic plane wave 
components, performing the extrapolation separately for each component, and superimposing the results. Consider one such upward-traveling harmonic component within the $l$ th layer. It can be written as

$$
p_{l}=P_{l}\left(k_{x}, \omega\right) \exp \left(j\left(k_{x} x+k_{l, z}\left(z-z_{l-1}\right)-\omega t\right)\right)
$$

where $\omega$ is the angular frequency, $k_{x}$ and $k_{l, z}$ are components of the wave number vector in the $l$ th layer, and $P_{l}\left(k_{x}, \omega\right)$ is the complex amplitude of the component. Here, we have used Snell's law that states that $k_{x}$ is preserved at a refracting interface that is parallel to the $x$-axis. Thus, we write $k_{x}$ without a layer index, $l$. Note that writing the component's z-dependence as a function of the difference $z-z_{l-1}$ instead of $z$ merely serves the purpose of simplifying the notation in the following.

For a point belonging to the $l$ th layer, the 2-D scalar lossless wave equation states that

$$
\left[\frac{\partial^{2}}{\partial x^{2}}+\frac{\partial^{2}}{\partial z^{2}}-\frac{1}{c_{l}^{2}} \frac{\partial^{2}}{\partial t^{2}}\right] p(x, z, t)=0
$$

By inserting (1) into (2) we get

$$
\left(-k_{x}^{2}-k_{l, z}^{2}+\frac{\omega^{2}}{c_{l}^{2}}\right) p_{l}=0
$$

and for a non-trivial solution, the dispersion relation

$$
k_{x}^{2}+k_{l, z}^{2}=\frac{\omega^{2}}{c_{l}^{2}}
$$

must be fulfilled. From this, we can obtain $k_{l, z}$ as a function of $\omega, c_{l}$, and $k_{x}$. If we consider only propagating (nonevanescent) waves, we have

$$
k_{l, z}=-\sqrt{\frac{\omega^{2}}{c_{l}^{2}}-k_{x}^{2}}, \quad \text { with } \frac{\omega^{2}}{c_{l}^{2}} \geq k_{x}^{2}
$$

where we have used the fact that $k_{l, z}$ is negative for an upward-traveling wave component.

By inserting (5) into (1), we obtain

$$
p_{l}=P\left(z, k_{x}, \omega\right) \exp \left(j\left(k_{x} x-\omega t\right)\right),
$$

with

$$
P\left(z, k_{x}, \omega\right)=P_{l}\left(k_{x}, \omega\right) \alpha_{l}\left(k_{x}, \omega, z-z_{l-1}\right),
$$

where $\alpha_{l}\left(k_{x}, \omega, \zeta\right)$ is defined as

$$
\alpha_{l}\left(k_{x}, \omega, \zeta\right)=e^{-j \zeta \sqrt{\left(\omega^{2} / c_{l}^{2}\right)-k_{x}^{2}}}
$$

To find $P_{l}\left(k_{x}, \omega\right)$, first consider layer $l=1$. Note that at $z=0$, the component $p_{1}$ is simply

$$
p_{1}=P_{1}\left(k_{x}, \omega\right) \exp \left(j\left(k_{x} x-\omega t\right)\right)
$$

and, because the exponent is the conjugate of the kernel in a 2-D Fourier transform over $x$ and $t$, the complex amplitude $P_{1}\left(k_{x}, \omega\right)$ must simply be the Fourier coefficient for the pair $\left(k_{x}, \omega\right)$ which we obtain by a 2 -D Fourier transformation of the field measured at $z=0$.

For a point within layer $l=2$, the field extrapolated to depth $z_{1}$ is used as the boundary condition defining the solution within the layer. This gives us the solution

$$
p_{2}=P\left(z, k_{x}, \omega\right) \exp \left(j\left(k_{x} x-\omega t\right)\right),
$$

with

$$
P\left(z, k_{x}, \omega\right)=P_{1}\left(k_{x}, \omega\right) \alpha_{1}\left(k_{x}, \omega, d_{1}\right) \alpha_{2}\left(k_{x}, \omega, z-z_{1}\right) .
$$

By proceeding in a similar way for the remaining layers, we find that the component can be extrapolated to a general point within layer $l$ using (6) with

$$
P\left(z, k_{x}, \omega\right)=P_{1}\left(k_{x}, \omega\right) \alpha_{l}\left(k_{x}, \omega, z-z_{l-1}\right) \prod_{q=1}^{l-1} \alpha_{q}\left(k_{x}, \omega, d_{q}\right)
$$

Note again that transmission losses at the interfaces are neglected. A correction for these can easily be included in the model, but for the purpose of imaging and with the relatively small angle of divergence for the transducer used in this work, such a correction is of limited value; see further comments in Appendix A.

The multiplication by $\alpha(\cdot)$ represents a phase shift, and it is from this relation that phase shift migration takes its name. Eq. (12) describes the relation between the 2-D Fourier transform of the field observed at $z=0$, and the 2-D Fourier transform of the field that would have been measured at $z \neq 0$, had the sensors been placed at that particular depth instead of $z=0$. Thus, $p(x, z, t)$ can be recovered by a 2-D inverse Fourier transform.

Consider now an exploding reflector at the particular depth, $z$, identical with the depth of a hypothetical sensor plane. Because the vertical distance between the sensor plane and the reflector then is zero, the contribution from the reflector will appear at $t=0$. Moreover, compared with any other observation time, the field originating from the reflector will be maximally concentrated in space at $t=0$. We realize that by simply reading out the field at time $t=0$ from $p(x, z, t)$ for all points at the same depth, $z$, we will obtain a horizontal image line that has optimal lateral resolution. Time $t=0$ is sometimes called the imaging condition for this scenario.

The inverse Fourier transform for obtaining $p(x, z, t=$ $0)$ is

$$
p(x, z, t=0)=\iint P\left(z, k_{x}, \omega\right) e^{j k_{x} x} \mathrm{~d} k_{x} \mathrm{~d} \omega
$$

where we have used the fact that $e^{-j \omega t}=1$ for $t=0$. The integral can be evaluated by first integrating $P\left(z, k_{x}, \omega\right)$ over $\omega$, followed by a 1-D inverse Fourier transform over $k_{x}$. 
Finally, note that the computation of $P\left(z, k_{x}, \omega\right)$ in (12) allows for an efficient recursive computation within each layer. If we have $P\left(z, k_{x}, \omega\right)$, we can obtain $P\left(z+\Delta z, k_{x}, \omega\right)$ with $z$ and $z+\Delta z$ both belonging to layer $l$, using

$$
P\left(z+\Delta z, k_{x}, \omega\right)=P\left(z, k_{x}, \omega\right) \alpha_{l}\left(k_{x}, \omega, \Delta z\right)
$$

so if we choose an image grid that is regular in $z$, the factor $\alpha_{l}\left(k_{x}, \omega, \Delta z\right)$ will be the same for each image line within that layer, meaning that it can be pre-calculated and stored in a lookup table to speed up the computations.

\section{B. Adaptations to the Case of Pulse-Echo Measurements}

The phase shift migration equations were derived assuming an exploding reflector model and this model must be adapted to the monostatic case. The sound paths in the pulse-echo measurements are twice as long as those in an exploding reflector scenario, so the pulse-echo measurements can be translated into an analogous exploding reflector scenario by simply dividing the physical sound speed by two.

As a consequence, the phase shift factor should, for the monostatic case, be redefined as

$$
\alpha_{l}\left(k_{x}, \omega, \zeta\right)=\exp \left\{-j \zeta \sqrt{\frac{4 \omega^{2}}{c_{l}^{2}}-k_{x}^{2}}\right\} .
$$

Another deviation from this theory is that the field measurements are influenced by the transducer characteristics. The electro-acoustical properties of the transducer affect the measured signal both at transmission and reception and the overall effect is that the received signals become band-pass filtered and, more importantly, phase delayed. If these delays are not compensated for, the imaging condition $t=0$ will be poorly synchronized with the waves resulting from a scatter at $z$, and this will cause a deterioration of the lateral resolution.

A simple way to compensate for these phase delays is to correlate the received signals with the double path impulse response of the transducer ${ }^{2}$ which is the method chosen here. After the correlation, the maximum peak of an echo from a small reflector at a certain range will appear correctly synchronized with the two-way travel time predicted by the range. A good approximation of this impulse response used in the correlation is obtained by measuring the response from a reflector in the far field parallel to the transducer surface [22] and the correlation is conveniently performed in the frequency domain for each acquired signal, $s\left(x_{n}, z=0, t\right)$, as

$$
P_{C}\left(x_{n}, z=0, \omega\right)=S\left(x_{n}, z=0, \omega\right) H^{*}(\omega)
$$

where $S\left(x_{n}, z=0, \omega\right)$ is the Fourier transform of $s\left(x_{n}, z\right.$ $=0, \omega)$ and $H^{*}(\omega)$ is the complex conjugate of the Fou-

\footnotetext{
${ }^{2}$ The double path impulse response is a convolution between the electro-mechanical impulse response in transmit and the mechanical-electrical in receive.
}

rier transform of the double path transducer impulse response.

We should note yet another deviation between reality and the assumptions used in the derivation. Because the diameter of the planar transducer is larger than the involved wavelengths, only the field from a limited set of angles centered around the normal to the transducer surface is sensed. Thus, the field that is processed is not the true field at the scanning surface but a spatially filtered version of it. Because the resolution in the reconstructed image is inversely proportional to the spatial bandwidth, and this bandwidth, in turn, is inversely proportional to the aperture of the sensor, the resolution will be proportional to the aperture. This is in accordance with the results presented in [23], showing that the resolution in synthetic aperture imaging is approximately $D / 2$ where $D$ is the aperture of the sensor.

\section{Summary of the Algorithm}

The algorithm summarized in this section attempts to reconstruct an $M \times N$ image from points on a regular grid in a region of interest that is defined as the rectangle $z \in\left[z_{\min }, z_{\max }\right], x \in\left[x_{\min }, x_{\max }\right]$. In the following, we let $z^{m}$ denote the $z$-coordinates of the $m$ th horizontal image line. ${ }^{3}$ These lines are separated by a distance $\Delta z=\left(z_{\max }\right.$ $\left.-z_{\min }\right) /(M-1)$ and we have $z^{m}=z_{\min }+(m-1) \Delta z$. In a similar way the $N$ vertical image lines are separated by the spatial sampling distance $\Delta x$.

Let $p_{C}\left(x_{n}, z=0, t\right)$ denote a signal acquired at the transducer position $x_{n}$, which has been correlated to the transducer impulse response as described in (16). The phase shift migration algorithm used in this work can be summarized as follows:

1) Apply a 2-D fast Fourier transform (fft) to the data: $P_{1}\left(k_{x}, \omega\right)=P\left(0, k_{x}, \omega\right) \leftarrow \mathrm{fft}_{t x}\left\{p_{C}\left(x_{n}, z=0, t\right)\right\}$, where the subscripts on the fft indicate which variables are transformed.

2) If $z_{\min }$ is not zero, use (12) to compute $P\left(z_{\min }, k_{x}, \omega\right)$.

3) Set $z^{1}=z_{\min }$ and do the following for all image lines $m=1$ to $M$ :

Compute $p\left(x, z^{m}, t=0\right)$ by first summing $P\left(z^{m}, k_{x}, \omega\right)$ over $\omega$,

$$
P\left(z^{m}, k_{x}\right)=\sum_{\omega} P\left(z^{m}, k_{x}, \omega\right),
$$

followed by a 1-D inverse fast Fourier transform:

$$
p\left(x, z^{m}, t=0\right) \leftarrow \operatorname{ifft}_{x}\left\{P\left(z^{m}, k_{x}\right)\right\},
$$

and assign $p\left(x, z^{m}, t=0\right)$ to the $m$ th horizontal image line.

\footnotetext{
${ }^{3}$ Please note the distinction between superscripts identifying the image lines and the subscripts used to identify layer interface coordinates
} 


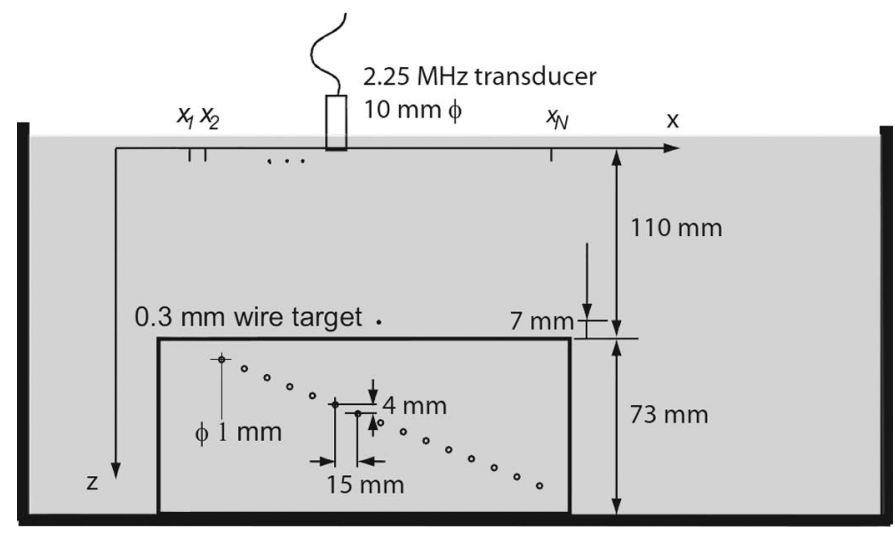

Fig. 2. Schematic setup for immersion testing of the copper block containing side-drilled holes. The ultrasonic transducer was scanned in the $x$-direction and pulse-echo measurements were performed at discrete locations $x_{1}, \ldots, x_{N}$ that were separated by $\Delta x=1 \mathrm{~mm}$.

b) If $z^{m}$ and $z^{m+1}$ both belong to the same layer, $l$, use $(14)$ to compute $P\left(z^{m+1}, k_{x}, \omega\right)$ for all values of $k_{x}$ and $\omega$. Else, if $z^{m}$ belongs to $l$ and $z^{m+1}$ belongs to $l+1$, compute $P\left(z^{m+1}, k_{x}, \omega\right)$ in two steps as $P\left(z^{m+1}, k_{x}, \omega\right)=P\left(z_{l}, k_{x}, \omega\right) \alpha_{l+1}\left(k_{x}, \omega, z^{m+1}-z_{l}\right)$ with $P\left(z_{l}, k_{x}, \omega\right)=P\left(z^{m}, k_{x}, \omega\right) \alpha_{l}\left(k_{x}, \omega, z_{l}-z^{m}\right)$.

Again note that the phase factors $\alpha(\cdot)$, appearing in steps 2 and 3(b), should be for the monostatic case as defined in (15).

In these algorithmic steps, variables without subscript indices should be considered over their full range. As an example, $p(x, z=0, t)$ is represented by an $N_{x} \times N_{t}$ matrix where $N_{x}$ and $N_{t}$ are the number of grid points in $x$ and the number of time samples used in the processing. Because going from continuous to discrete data may introduce aliasing problems, zero-padding is generally required to avoid this. See Appendix B for a discussion on aliasing issues in both the temporal and spatial domains.

\section{EXPERIMENTS}

Two experiments were performed. The first demonstrated the method's ability to treat a scenario with scatterers residing in two layers having different sound speeds. This experiment, which is presented in Section III-A, was performed with a copper block immersed in water and containing several side-drilled holes (SDHs). A wire target was placed in the water in front of the block, creating a scenario with scatterers present both in the slow water media, with $c_{\mathrm{H} 2 \mathrm{O}}=1482 \mathrm{~m} / \mathrm{s}$, and in the fast copper media, with $c_{\mathrm{Cu}}=4660 \mathrm{~m} / \mathrm{s}$. The second experiment demonstrated how the method can be used with immersion test data to improve the resolution in $\mathrm{C}$-scans and improve the amplitude contrast between isolated reflectors and the contributions from grain scattering. In this experiment, presented in Section III-B, the test object was a copper block with flat bottom holes (FBHs).

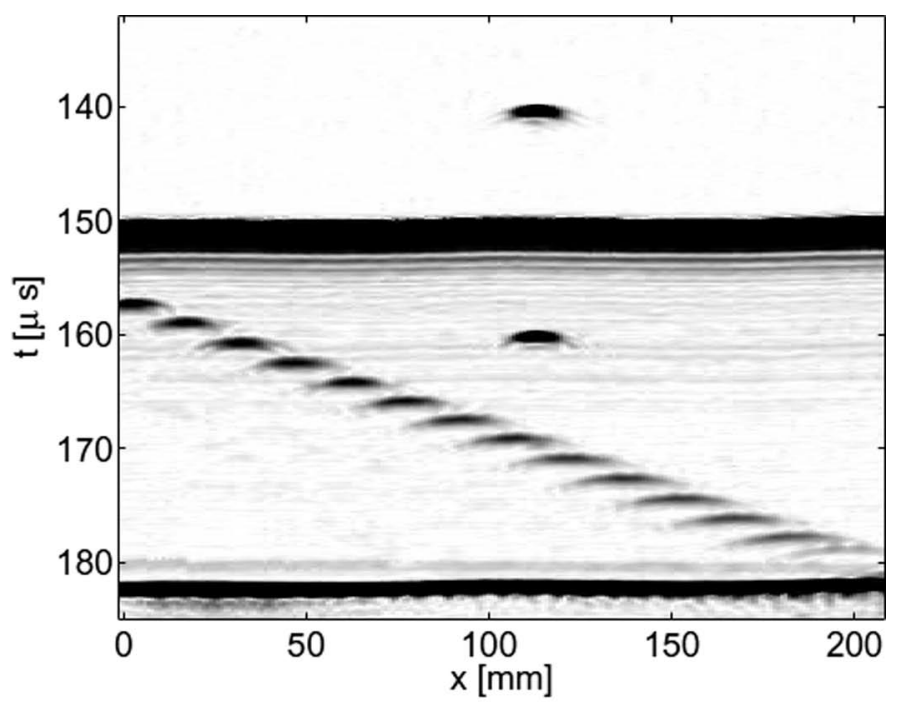

(a)

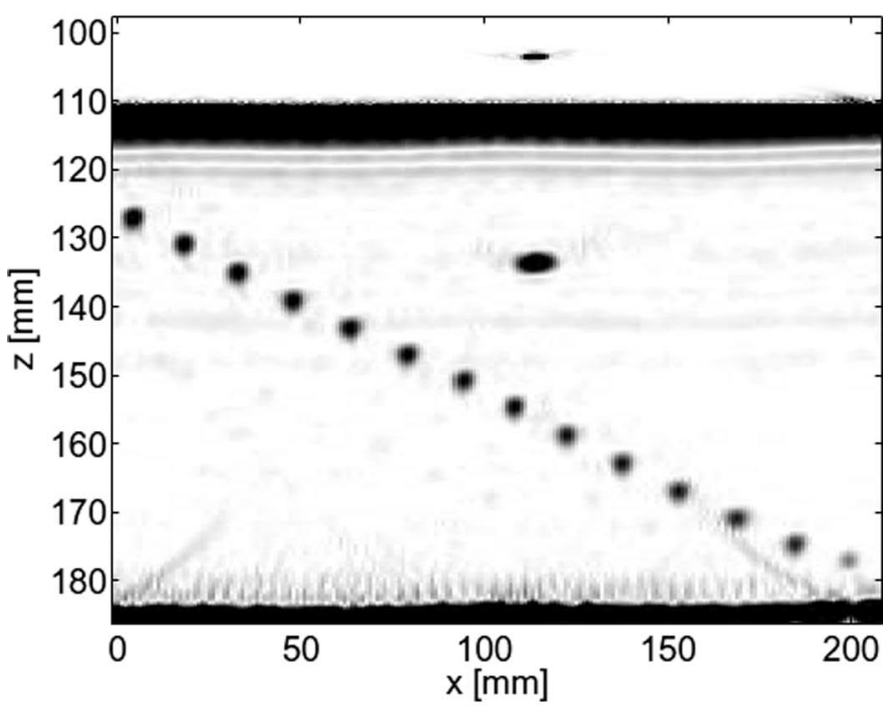

(b)

Fig. 3. (a) The B-scan from the copper block with side-drilled holes and with a wire target in front of the upper surface. (b) Result from phase shift migration.

\section{A. Immersion Measurements of Copper Block With SDHs and a Wire Target in Water}

The immersion test setup used in the first experiment is shown in Fig. 2. A planar circular 2.25-MHz transducer from Panametrics with $10 \mathrm{~mm}$ diameter was scanned along the $x$-axis and pulse-echo measurements were acquired at positions $x_{1}, \ldots, x_{210}$ that were separated by $\Delta x=1 \mathrm{~mm}$. The inspected object containing SDHs was placed with its front surface in the horizontal plane.

The acquired data are presented in Fig. 3(a) as an envelope B-scan, obtained by Hilbert transformation of the raw data. The front surface echo is seen at approximately $t=150 \mu \mathrm{s}$ corresponding to the water path of approximately $110 \mathrm{~mm}$, and the wire target can be seen at $x=$ $110 \mathrm{~mm}$ at $t=140 \mu \mathrm{s}$. A secondary echo from the wire is also seen at $t=160 \mu \mathrm{s}$ at the same scanning position. 


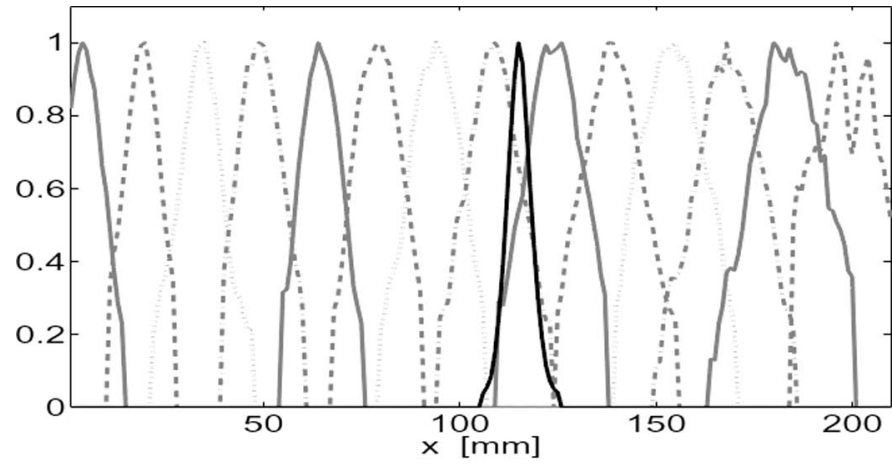

(a) B-scan

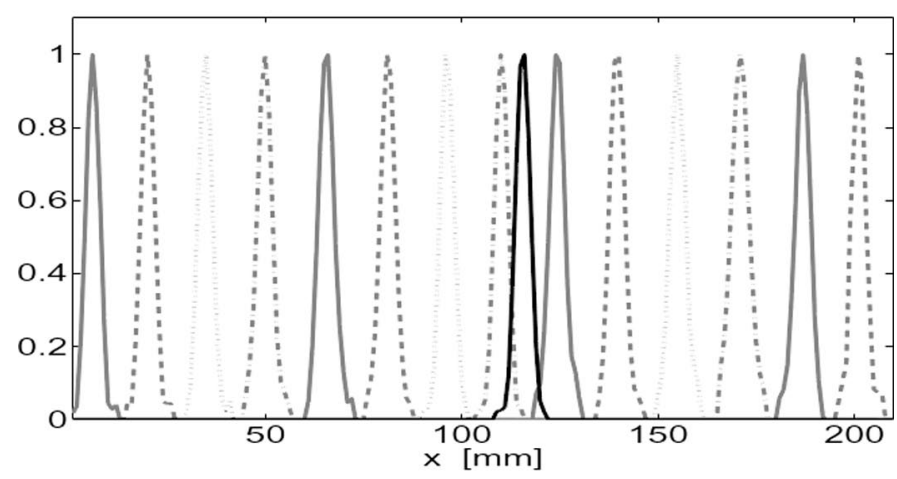

(b) Phase shift migration

Fig. 4. Profile plots of the side-drilled holes and the wire target in the original B-scan and the reconstructed image. The profiles of side-drilled holes are shown gray with various line styles to separate visually and the wire target is shown as a solid black line.

It corresponds to a transducer- $\mathrm{Cu}$-wire- $\mathrm{Cu}$-transducer sound path. The SDHs are seen as hyperbolic patterns at various depths.

In Fig. 3(b), the image reconstructed using phase shift migration is presented. In this image, in which the horizontal image lines are separated by $\Delta z=0.1 \mathrm{~mm}$, the responses from the SDHs are concentrated to small spots and the same holds for the wire target. Note that a geometrical correction is obtained through the migration because the method takes into account the different sound speeds at different layers. Thus, the wire target's distance between to the front surface of the block can be correctly measured in the image to be $7 \mathrm{~mm}$. Because of the shorter wavelength in water, the wire target has a better vertical resolution than the SDHs in the copper block. Note also that the lateral resolution of the SDHs is approximately uniform throughout the entire object.

The diffuse spot centered at $x=110 \mathrm{~mm}$ and $z=$ $132 \mathrm{~mm}$ corresponds the previously mentioned double reflection $\mathrm{Cu}$-wire- $\mathrm{Cu}$. Because multiple reflections are not taken into account in the development of the method, such echoes generally lead to blurred artifacts such as the one seen here.

The lateral resolution can be further examined in Fig. 4, where local profile plots for the SDHs as well as the wire target are shown both for the B-scan and the recon-

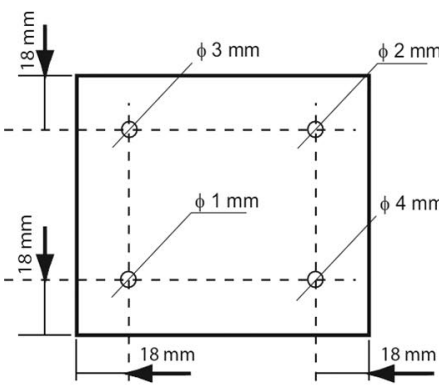

Bottom view

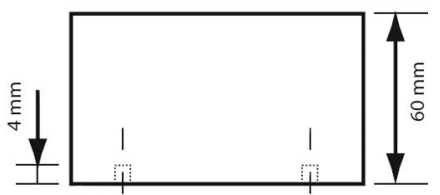

Side view
Fig. 5. Copper block with four flat bottom holes.

structed image. These profiles were obtained by calculating the maximum amplitudes within a depth interval covering the target of interest and projecting the values onto the $x$-axis. For ease of comparison, the profiles have been normalized to the same maximum amplitudes.

Inspection of the profile plots confirms the conclusion that the lateral resolution in the reconstructed image is practically independent of the depth. This holds also for the wire target which is surrounded by a medium with a different sound velocity than the SDHs. If we define the resolution to be the length of the cross section at $50 \%$ of the maximum of the amplitude, we get a resolution that is approximately $5 \mathrm{~mm}$ for both the holes and the wire target. This is in accordance with the results for synthetic aperture imaging [23], stating the resolution to be approximately $D / 2$ for a sensor with an aperture of $D$. Here we have $D=10 \mathrm{~mm}$.

\section{B. Immersion Test of Copper Block With FBHs}

In the second experiment, a volume scan of a copper block with FBHs was performed. The dimensions of the block are shown in Fig. 5. The purpose of the experiment was to illustrate the improvements in detectability and lateral resolution that can be achieved through the method. The block had a grainy structure that caused both strong back scattering noise as well as sound attenuation and the responses of the FBHs were therefore relatively difficult to detect in standard B-scans. Only the 4-mm diameter FBH gave a response strong enough to be easily detected in those images.

Figs. 6 and 7 show two examples of envelope B-scans from the data set along with the corresponding reconstructed images. Fig. 6 shows an extracted B-scan over the $1-\mathrm{mm}$ and $4-\mathrm{mm}$ FBHs. The $1-\mathrm{mm}$ hole is not seen at all, but the response from the $4-\mathrm{mm} \mathrm{FBH}$ can be seen at an interval around $x=82 \mathrm{~mm}$ and $t=82 \mu \mathrm{s}$. Note that the width of the response is approximately $15 \mathrm{~mm}$. In the corresponding reconstructed image, the $4-\mathrm{mm} \mathrm{FBH}$ at around $z=97 \mathrm{~mm}$ is reduced in size significantly.

Fig. 7 shows an extracted B-scan over the 2-mm and 3-mm holes, and we can see weak responses from these holes at around $t=82 \mu \mathrm{s}$, for $x$ around $18 \mathrm{~mm}$ and $80 \mathrm{~mm}$, respectively. The $3 \mathrm{~mm}$ and $2 \mathrm{~mm} \mathrm{FBHs}$ at around $z=$ $97 \mathrm{~mm}$ in the corresponding reconstructed image are bet- 


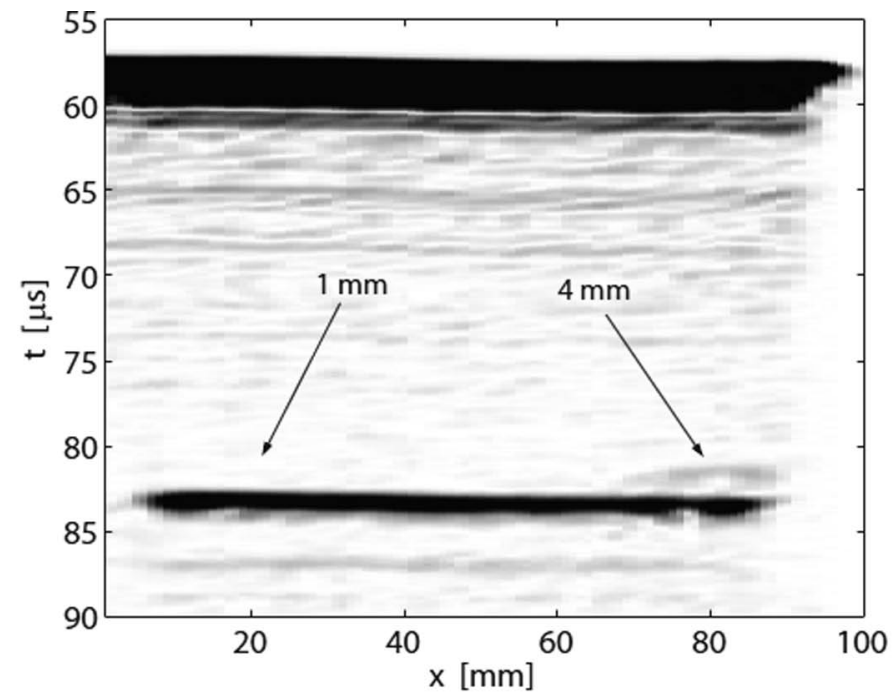

(a)

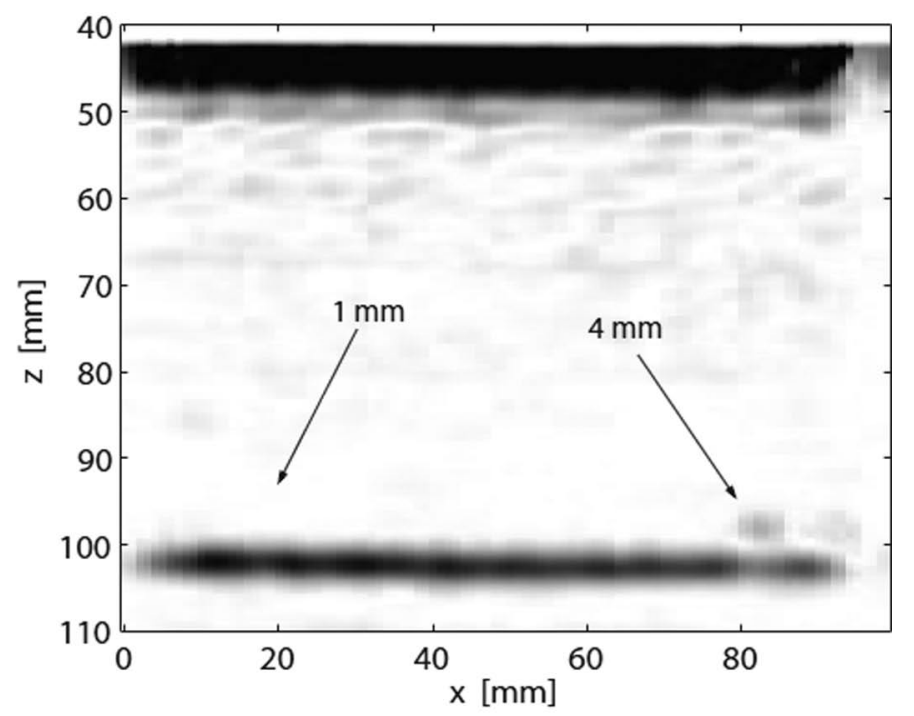

(b)

Fig. 6. (a) Original B-scan acquired at $y=18 \mathrm{~mm}$ passing over the $1-\mathrm{mm}$ and 4-mm flat bottom holes. (b) Image resulting from phase shift migration. The response of the $1-\mathrm{mm}$ flat bottom hole is too weak to be seen.

ter localized in the $x$-direction thus providing better conditions for extracting a C-scan.

C-scan images from the depth interval covering the $\mathrm{FBH}$ in the $\mathrm{Cu}$-block were extracted from the raw data as well as from the reconstructed images by projecting the maximum amplitude values within the interval onto the $x-y$ plane. The depth interval of interest corresponds to the interval $z \in[95,99] \mathrm{mm}$ if the water path is taken into account. In the C-scans that are presented in Fig. 8, the FBHs of diameters 2,3 , and $4 \mathrm{~mm}$ are visible in both $\mathrm{C}$-scans but the resolution is much improved in the $\mathrm{C}$ scan based on the migrated data. In particular, the 3-mm diameter $\mathrm{FBH}$ is, in the original $\mathrm{C}$-scan, partly masked by disturbances caused by early parts of the back wall echo entering the time window of interest. In the migrated $\mathrm{C}$ scan, these disturbances are less prominent and the true position of the FBH is more easily found.

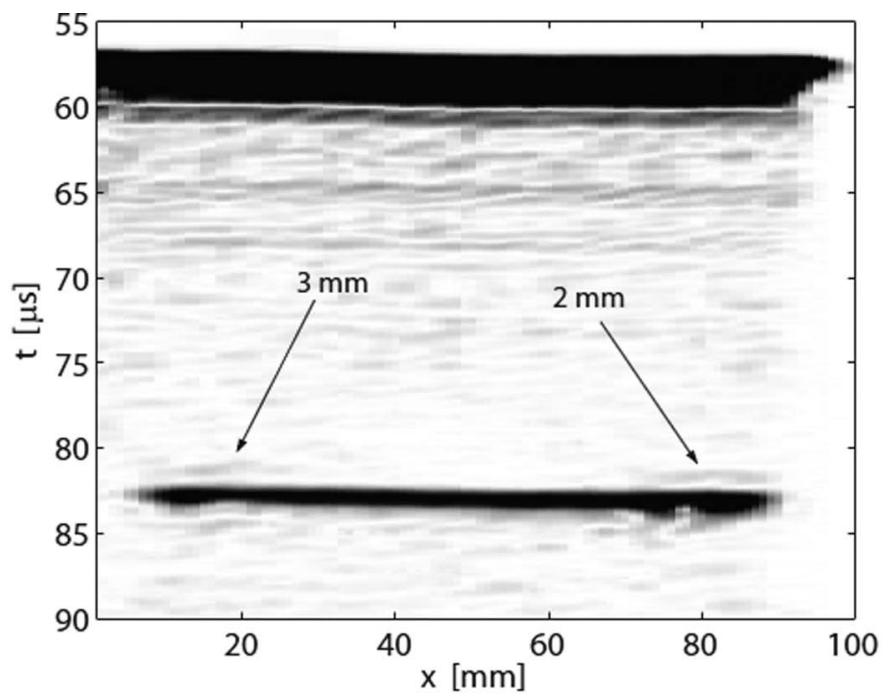

(a)

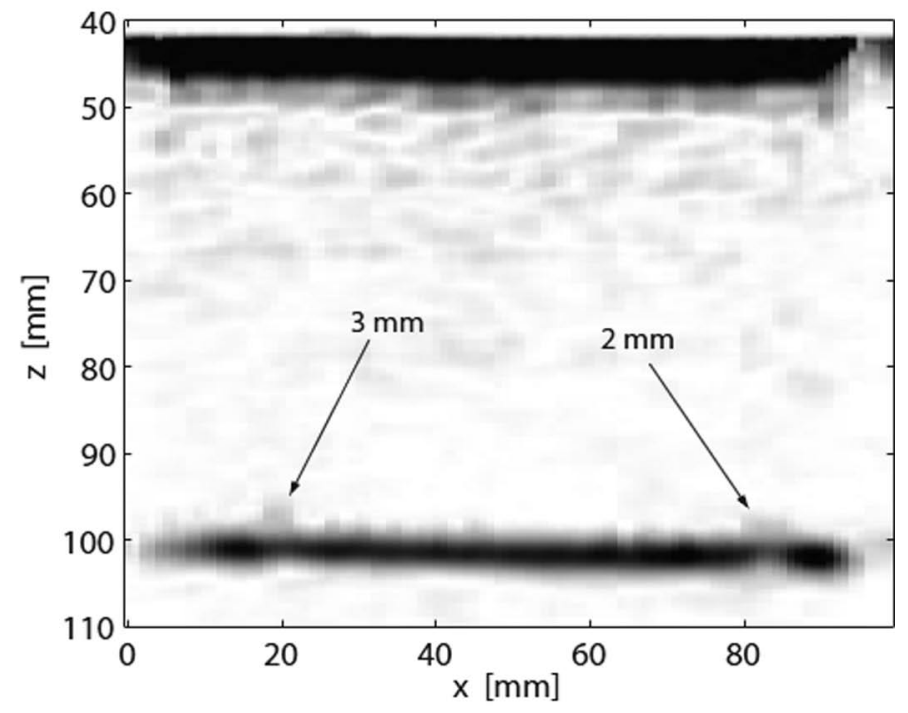

(b)

Fig. 7. (a) Original B-scan acquired at $y=63 \mathrm{~mm}$ passing over the $3-\mathrm{mm}$ and 2-mm flat bottom holes. (b) Image obtained by migration of the B-scan.

Note that the improvement in resolution only concerns the direction of migration; the migration has not significantly improved the resolution in the $y$-direction.

Both C-scans in Fig. 8 have been normalized with respect to their maximum pixel value providing a fair comparison of the noise level in the images, and we note that phase shift migration has improved the signal-to-noise ratio, giving better contrast.

\section{Conclusions And Discussion}

Phase shift migration has been proposed for ultrasonic imaging of objects immersed in water and the algorithm has been demonstrated to correctly treat media with different sound speeds and to yield images with an improved lateral resolution under such conditions.

The experiment with the copper block with SDHs showed that the lateral resolution in the reconstructed 


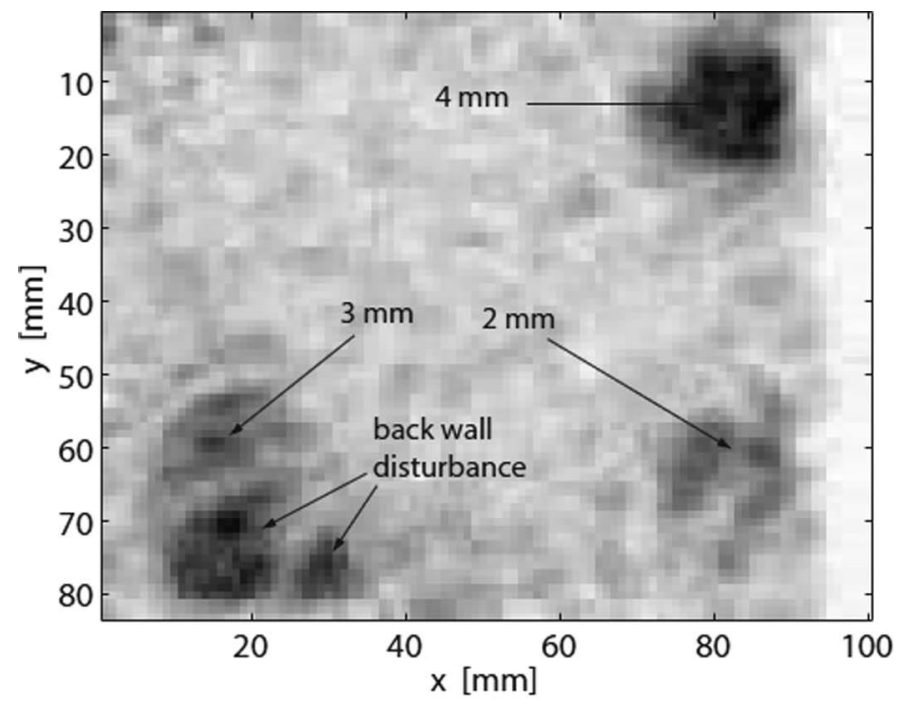

(a)

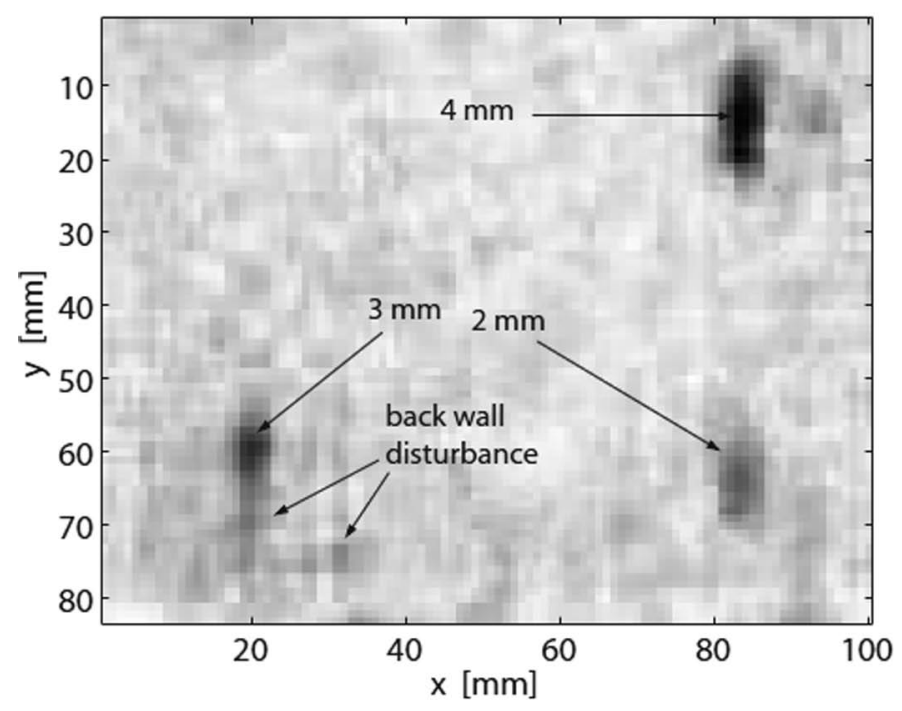

(b)

Fig. 8. C-scan based on (a) raw data and (b) migrated data. Note that the $\mathrm{C}$-scans are seen from a top view with the 4-mm diameter flat bottom hole seen at the upper-right corner.

image is independent of depth to a good approximation. It was also demonstrated using data from a block with FBHs that the phase shift migration, along with the resolution improvement, helps in improving the amplitude contrast between isolated reflectors and the contributions from grain scattering, and thus is a useful tool for the detection of defects that are buried deeply in grainy materials.

The method considers longitudinal propagation only and any contribution associated with shear waves are neglected. Two facts motivate this approximation: First, the velocity of shear waves is around half the velocity of the longitudinal waves, which means that the contributions from the two modes appear well separated in time, at least for deep reflectors. Second, the echo transmittance for shear waves is very small compared with the longitudinal. See Appendix A for numerical examples of the relative strengths of the mode components for the experiments presented in the paper.
The algorithm is implemented using fast Fourier transform routines and the current implementation allows processing of the B-scans that takes less time than the data acquisition. As an example, the acquisition time of the 1325 $\times 210$ B-scan used in the experiments presented in Section III-A was approximately $3 \mathrm{~min}$, whereas the processing time was $3.5 \mathrm{~s}$ on a dual core $2.80 \mathrm{GHz}$ laptop. Finally, we note that the method can be straightforwardly extended to processing of 3-D data, thus allowing for simultaneous improvement in both of the lateral dimensions.

\section{Appendix A: \\ Comments on Transmission Losses at Layer INTERFACES}

In the modeling, transmission losses at the layer interfaces were neglected and this will cause errors in the computed field as it is backpropagated through an interface. We should note, however, that as long as the effect of the transmission loss can be well approximated by a constant scaling, independent of angle of incidence and thus independent of $k_{x}$, the resulting effect on the reconstructed image will merely be an amplitude scaling that will be constant within each layer. Such a scaling will not influence the lateral resolution.

The circular transducer used in the experiments had a diameter of $D=10 \mathrm{~mm}$, a center frequency $F_{\mathrm{c}}=2.25 \mathrm{MHz}$, and bandwidth of approximately $\Delta F=1.5 \mathrm{MHz}$. Thus, the lowest frequency at which the transducer operated was $1.5 \mathrm{MHz}$, which yields a maximum wavelength of approximately $\lambda=1 \mathrm{~mm}$ in water. The maximum beam divergence is then given by [24] $\theta=\arcsin (1.22 \lambda / D) \approx 0.122$ radians or $7^{\circ}$. Thus, we know that the sound enters into the copper object with angle of incidence of at most $7^{\circ}$. The echo transmittances [24] for longitudinal-longitudinal waves at a water-copper interface calculated for angles between $0^{\circ}$ and $7^{\circ}$ vary between 0.1345 for $0^{\circ}$ and 0.1286 for $7^{\circ}$. Thus, the difference is maximally $6 \%$ and we can, in the experiments presented in this paper, safely approximate them as being constant for all angles of interest.

Furthermore, the echo transmittance for transversal waves appearing as a result of mode conversion at the boundary is less than $7 \%$ of the echo transmittance of longitudinal waves at $7^{\circ}$ and approximately $3 \%$ at $4.7^{\circ}$, which is the beam divergence at the center frequency. As a consequence, the contribution of the transversal waves to the final image will be very weak and this motivates neglecting these waves in the processing.

\section{Appendix B:}

\section{Comments on the Use of the Discrete Fourier Transform and Aliasing}

All Fourier transforms involved in the algorithm are performed using discrete data and the fast Fourier transform has been used for all calculations. Aliasing may then 
appear both spatially and temporally. The temporal sampling must fulfil the Nyquist criterion, stating that the sampling frequency must at least be twice that of the highest frequency appearing in the signals. This requirement is fulfilled with a large margin in the experiments presented below; the sampling frequency was $25 \mathrm{MHz}$, whereas the center frequency of the transducer was $2.25 \mathrm{MHz}$ and with a bandwidth of about $1.5 \mathrm{MHz}$.

The spatial sampling of the $x$-axis is more critical. In its conventional form, in which a scenario with constant velocity and point-like transducers are considered, the spatial sampling theorem states that the distance between two sampling points on the $x$-axis, $\Delta x$, should be separated by no more than $\lambda_{\min } / 4$ for pulse-echo measurements where $\lambda_{\text {min }}$ is the minimum wavelength appearing in the measurements. As an example, if we use a transducer with an upper frequency of $3 \mathrm{MHz}$ for the measurements in water, having a velocity of $1500 \mathrm{~m} / \mathrm{s}$, we have $\lambda_{\min }=0.5 \mathrm{~mm}$ and $\Delta x<0.125 \mathrm{~mm}$, which is a quite small separation that will lead to impractically long data acquisition times if large areas are to be scanned.

The requirement $\Delta x<\lambda_{\min } / 4$ is, however, quite a bit too pessimistic and does not take into account the transducer's directivity. If this is done, we arrive at the much less restrictive result [25] stating that when a circular transducer of diameter $D$ is used, the spatial sampling must be performed at steps no longer than $\Delta x<D / 4$. For a transducer of $10 \mathrm{~mm}$, we thus have that $\Delta x<2.5 \mathrm{~mm}$ which is fulfilled in our measurements.

Finally, aliasing can occur also at the inverse transform back to spatial coordinates. The Fourier coefficients are essentially connected to a function that is periodic both in $t$ and $x$ and especially the periodicity in $x$ may cause problems in the reconstruction. The problem can be avoided by using zero-padding of the measured data in the $x$-direction.

Consider scanning over a region-of-interest that has length $W_{x}$ in the $x$-direction and has a maximum depth of $z_{\max }$ with a transducer with divergence angle $\theta$. Say that $N_{x}$ is the number of samples used in the Fourier transform in the $x$-direction after zero-padding. According to [26], the minimum $N_{x}$ required to avoid aliasing is then approximately given by

$$
N_{x, \min }=\left\lceil\frac{\frac{W_{x}}{2}+\frac{z_{\max }}{\cot (\theta)}+\frac{N \Delta x}{2}}{\Delta x}\right\rceil,
$$

where $N$ and $\Delta x$ are the number of acquired A-scans and the scanning step, respectively, and \lceil\rceil denotes the ceiling operator.

\section{REFERENCES}

[1] D. W. Prine, "Synthetic aperture ultrasonic imaging," in Proc. Engineering Applications of Holography Symp., Jul. 1972, pp. 287-288.

[2] K. Nagai, "Fourier domain reconstruction of synthetic focus acoustic imaging system," Proc. IEEE, vol. 72, no. 6, pp. 748-749, 1984.

[3] K. Langenberg, M. Berger, T. Kreutter, and K. Mayer, "Synthetic aperture focusing technique signal processing," NDT Int., vol. 19, no. 3, pp. 177-189, Jun. 1986.
[4] K. Mayer, R. Marklein, K. Langenberg, and T. Kreutter, "Three dimensional imaging system based on fourier transform synthetic aperture focusing technique," Ultrasonics, vol. 28, no. 4, pp. 241-255, 1990.

[5] L. Busse, "Three-dimensional imaging using a frequency domain synthetic aperture focusing technique," IEEE Trans. Ultrason. Ferroelectr. Freq. Control, vol. 39, no. 2, pp. 174-179, 1992.

[6] Z. D. Qin, A. Tauriainen, J. Ylitalo, E. Alasaareal, and W. Lu, "Frequency domain compensation for inhomogeneous layers in ultrasound holography," IEEE Trans. Ultrason. Ferroelectr. Freq. Control, vol. 36, no. 1, pp. 73-78, Jan. 1989.

[7] R. Stolt, "Migration by Fourier transform," Geophysics, vol. 43, no. 1, pp. 23-48, Feb. 1978.

[8] T. Stepinski, "An implementation of synthetic aperture focusing techniques in frequency domain," IEEE Trans. Ultrason. Ferroelectr. Freq. Control, vol. 54, no. 7, pp. 1399-1408, Jul. 2007.

[9] S. Doctor, T. Hall, and L. Reid, "Saft-the evolution of a signal processing technology for ultrasonic testing," NDT Int., vol. 19, no. 3, pp. 163-167, 1986.

[10] E. Wennerström and T. Stepinski, "Model-based correction of diffraction effects of the virtual source element," IEEE Trans. Ultrason. Ferroelectr. Freq. Control, vol. 54, no. 8, pp. 1614-1622, Aug. 2007.

[11] J. A. Johnson and A. Barna, "The Effects of Surface Mapping Correction with Synthetic-Aperture Focusing Techniques on Ultrasonic Imaging," IEEE Trans. Sonics Ultrason., vol. SU-30, no. 5, pp. 283294, Sept. 1983.

[12] H. G. Kraus, "Generalized synthetic aperture, focused transducer, pulse-echo, ultrasonic scan data processing for nondestructive inspection," Ultrasonics, vol. 21, no. 1, pp. 11-18, Jan. 1983.

[13] A. Shlivinski and K. Langenberg, "Defect imaging with elastic waves in inhomogeneous-anisotropic materials with composite geometries," Ultrasonics, vol. 46, no. 1, pp. 89-104, 2007.

[14] M. Taner and F. Koehler, "Velocity spectra-digital computer derivation and applications of velocity functions," Geophysics, vol. 34, no. 6, pp. 859-881, Dec. 1969.

[15] M. A. Haun, D. L. Jones, and W. D. O'Brien, Jr., "Adaptive focusing through layered media using the geophysical 'time migration' concept," in Proc. IEEE Ultrasonics Symp., 2002, vol. 2, pp. 1635-1638.

[16] J. Gazdag, "Wave equation migration with the phase-shift method," Geophysics, vol. 43, no. 7, pp. 1342-1351, Dec. 1978.

[17] J. F. Claerbout, Imaging the Earth's Interior. Cambridge, MA: Blackwell Scientific, 1985.

[18] J. Gazdag and P. Sguazzero, "Migration of seismic data by phase shift plus interpolation," Geophysics, vol. 49, no. 2, pp. 124-131, Feb. 1984.

[19] F. Bertora, P. Pellegretti, A. Questa, C. Parodi, and A. Trucco, "An alternative frequency domain beamforming," in Proc. IEEE Ultrasonics Symp., 2004, vol. 3, pp. 1749-52.

[20] D. Loewenthal, L. Lu, R. Roberson, and J. Sherwood, "The wave equation applied to migration," Geophys. Prospect., vol. 24, no. 2, pp. 380-399, Jun. 1976.

[21] W. Schneider, "Integral formulation for migration in two and three dimensions," Geophysics, vol. 43, no. 1, pp. 49-76, Feb. 1978.

[22] D. Cassereau, D. Guyomar, and M. Fink, "Time deconvolution of diffraction effects-application to calibration and prediction of transducer waveforms," J. Acoust. Soc. Am., vol. 84, no. 3, pp. 1073-1085, Sep. 1988.

[23] L. Cutrona, "Comparison of sonar system performance achievable using synthetic aperture techniques with the performance achievable by more conventional means," J. Acoust. Soc. Am., vol. 58, no. 2, pp. 336-348, 1975.

[24] J. Krautkramer and H. Krautkramer, Ultrasonic Testing of Materials. New York, NY: Springer, 1990.

[25] M. Soumekh, Synthetic Aperture Radar Signal Processing with Matlab Algorithms. New York, NY: Wiley, 1999.

[26] K. Gu, G. Wang, and J. Li, "Migration based SAR imaging for ground penetrating radar systems," IEE Proc., Radar Sonar Navig., vol. 151 , no. 5, pp. 317-325, Oct. 2004.

Tomas Olofsson was born 1968 in Sandviken, Sweden. He received his M.Sc. degree in engineering physics in 1994 and the Ph.D. degree in signal processing in 2000, both from Uppsala University. He is currently working as an associate professor in the Signals and Systems group, Uppsala University, Sweden. His research concerns inference problems, in particular inverse problems in ultrasonics. 